\title{
Synergy and complementarity between neutrino physics and low-energy intensity frontiers
}

\author{
Ana M. Teixeira* \\ Laboratoire de Physique de Clermont, CNRS/IN2P3 - UMR 6533, \\ Campus des Cézeaux, 4 Av. Blaise Pascal, F-63178 Aubière Cedex, France \\ E-mail: ana.teixeira@clermont.in2p3.fr
}

\begin{abstract}
Massive neutrinos and leptonic mixings have provided the first evidence of flavour violation in the lepton sector, opening a unique gateway to many new phenomena. Among the latter, one finds processes violating lepton number, charged lepton flavours, or even the universality of lepton flavours. These very rare transitions can be studied in high-intensity facilities, and if observed, constitute a clear sign of New Physics. After a brief review of the experimental status of dedicated searches, we comment on the prospects of several well-motivated models of neutrino mass generation to several of the above mentioned observables, also discussing how the interplay of high-intensity observables and neutrino data can shed light on the underlying New Physics model.
\end{abstract}

The 19th International Workshop on Neutrinos from Accelerators-NUFACT2017

25-30 September, 2017

Uppsala University, Uppsala, Sweden

\footnotetext{
* Speaker.
} 


\section{Introduction}

Neutrino oscillations provided the first confirmation that the Standard Model (SM) should be extended; eversince, the quest for the new physics (NP) model accounting for neutrino masses and mixings has become one of the most active quests in particle physics. There is a vast array of wellmotivated SM extensions, relying on additional fields, extended gauge groups, or even complete NP frameworks - all capable of successfully accommodating neutrino oscillation data. Fortunately, massive neutrinos and leptonic mixings offer a true gateway to many experimental signals that are either forbidden or extremely suppressed in the SM; these include charged lepton flavour violation (cLFV), lepton number violation (LNV), contributions to lepton dipole moments, among many others. The interplay of oscillation data with the results of the searches for these rare processes, which are being actively looked for at numerous high-intensity facilities, may then allow to shed light on, and hopefully identify, the underlying model of neutrino masses and mixings.

In the original formulation of the SM, neutrinos are strictly massless and lepton number(s) are conserved; leptonic electric dipole moments (EDMs) are generated at 4-loop level, and their value is tiny $\left(d_{e}^{\mathrm{CKM}} \leq 10^{-38} e \mathrm{~cm}\right)$. Minimal SM extensions in which Dirac $v$ masses are put by hand, and the leptonic mixing matrix $U_{\text {PMNS }}$ accounts for $v$ oscillation data, still preserve total lepton number; cLFV transitions can theoretically occur, but the smallness of neutrino masses strongly suppresses the branching fractions, rendering them unobservable. Likewise, and despite being generated at the 2-loop level, EDMs still remain beyond experimental sensitivity.

Reviews of the experimental status of numerous (rare) leptonic processes were conducted in the dedicated NUFACT2017 sessions, as well as in plenary presentations [1]; a full summary of current bounds, including electric and magnetic (anomalous) lepton moments can be found in [2].

A detailed discussion of the model-independent approach to constrain NP models based on the searches for the above mentioned observables has been done in [3]; in what follows, we focus the discussion on specific NP realisations - from simple, minimal SM extensions, to complete frameworks.

\section{SM extensions via sterile neutrinos}

Sterile fermions are an integral part of several well-motivated mechanisms of neutrino mass generation; before addressing the contributions of the latter constructions to different high-intensity observables (and discuss how the interplay of distinct signals can favour or exclude them), a first phenomenological - and convenient - approach consists in considering simple "toy models", in which the SM is extended by a single massive sterile state (possibly encoding the effects of a larger number of sterile states).

\subsection{Minimal " $3+1$ toy model”"}

Without any assumption on the mechanism of neutrino mass generation, this simple construction relies in extending the SM content via one massive heavy sterile state; the interaction and physical basis are related via a $4 \times 4$ unitary matrix $U$ (whose upper $3 \times 3$ block encodes left- 
handed leptonic mixings). The non-negligible active-sterile mixings are at the source of modified charged and neutral lepton currents, and hence of new contributions to many observables ${ }^{1}$.

For example, concerning EDMs, the new Majorana and Dirac phases induce non-vanishing contributions; in the presence of two non-degenerate sterile states (with masses between $100 \mathrm{GeV}$ and $100 \mathrm{TeV}$ ), one can have $\left|d_{e}\right| / e \geq 10^{-30} \mathrm{~cm}$, within future ACME sensitivity [ $\llbracket$ ], as displayed on the left panel of Fig. 1. Since the Majorana contribution is dominant, the interpretation of an EDM observation in such a minimal framework would suggest CP violating Majorana neutrinos, with potential implications for leptogenesis.
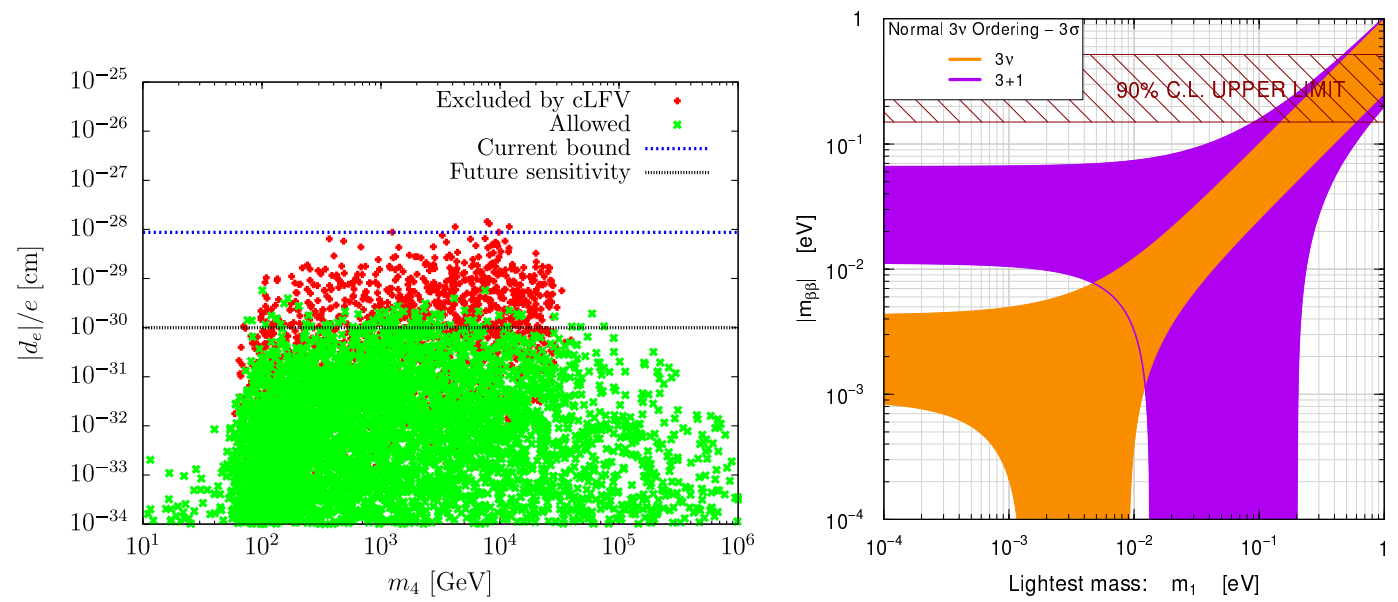

Figure 1: On the left, contributions to $\left|d_{e}\right| / e$ as a function of the sterile neutrino masses; from 帆. On the right, effective Majorana mass $m_{\beta \beta}$ as a function of the lightest (active) neutrino mass (normal ordering of the light $v$ spectrum); from [5].

Other than strongly impacting the prediction for $0 v 2 \beta$ decays (due to the augmented ranges for the effective mass in both normal and inverted ordering schemes, a future observation can no longer be straightforwardly associated with an inverted ordering [5], as visible on the right panel of Fig. 11), the sterile states can also be at the origin of LNV semileptonic tau and meson decays. If produced on-shell, sterile neutrinos can lead to a resonant enhancement of the LNV decay amplitudes, some processes already within experimental reach, as is the case of $\tau^{-} \rightarrow \mu^{+} \pi^{-} \pi^{-}$, or $K^{+} \rightarrow \ell_{\alpha}^{+} \ell_{\beta}^{+} \pi^{-}$ (see left panel of Fig. 2). A comprehensive study of such decays allows to infer bounds on all entries of a generalised definition of the effective Majorana mass matrix: with the exception of the $m_{v}^{\tau \tau}$ entry (whose bounds $\lesssim 10^{-2} \mathrm{GeV}$ strongly improve existing ones), all other entries are constrained to lie below $\lesssim 10^{-4} \mathrm{GeV}$ [6]. An example for $m_{v}^{\mu \mu}$ is displayed on Fig. 2.

Such a minimal construction also leads to important contributions to cLFV observables: in the $e-\mu$ sector, neutrinoless conversion in Nuclei (e.g. Aluminium) is one of the most sensitive observables (although for heavier nuclei, the Coulomb-enhanced decay of a muonic atom might be also competitive [7], see Fig. 3). For sterile states heavier than the electroweak scale, threebody decays receive the dominant contributions from $Z$-penguins, leading to a strong correlation between the corresponding cLFV decays. In turn, this not only allows to probe $\mu-\tau$ flavour

\footnotetext{
${ }^{1}$ In many of the subsequent numerical results, the additional physical parameters of the model were randomly sampled from the following intervals: $m_{4} \in\left[0.1-10^{5}\right] \mathrm{GeV}, 0 \lesssim \theta_{\alpha 4} \lesssim 2 \pi$ (and likewise for the CP violating phases).
} 

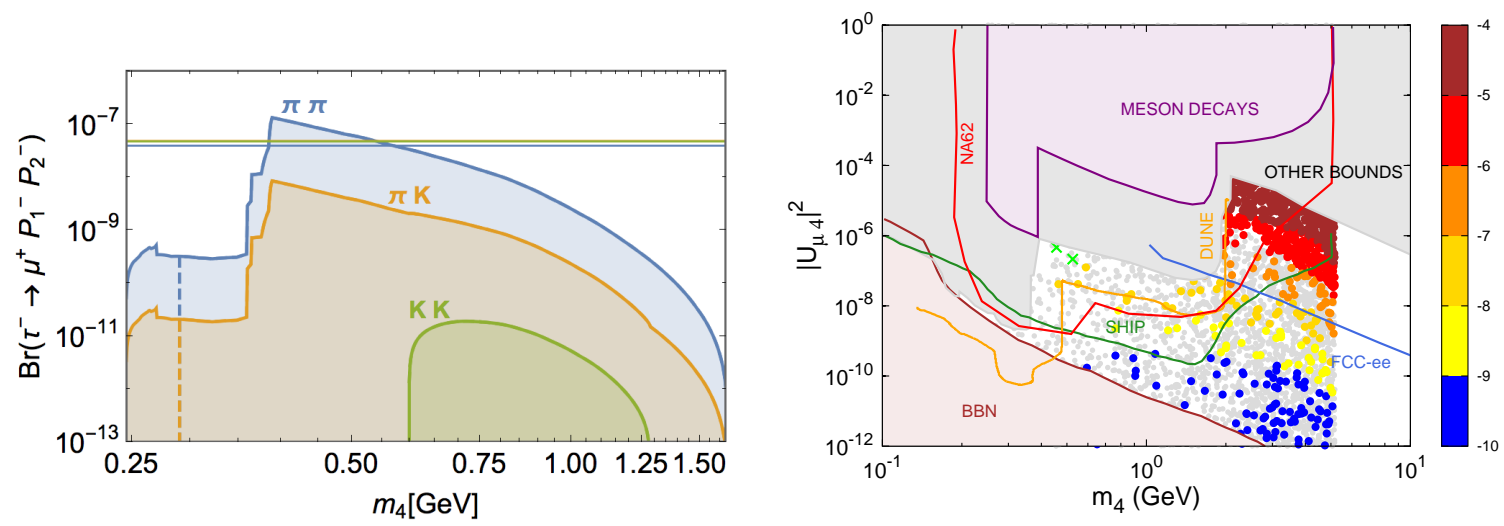

Figure 2: On the left, lines (surfaces) denoting the maximal (allowed) $\mathrm{BR}\left(\tau^{-} \rightarrow \mu^{+} P_{1}^{-} P_{2}^{-}\right.$) vs. the heavy neutrino mass, $m_{4}$. On the right, predictions for the effective mass, $\log m_{v}^{\alpha \beta}$, in the $\left(\left|U_{\mu 4}\right|^{2}, m_{4}\right)$ plane, as derived from LNV B-meson decays. Coloured surfaces and grey points denote excluded regimes. From [6].

violation beyond the reach of Belle II, but also to explore this minimal SM extension at several frontiers [8], as displayed in Fig. 3.
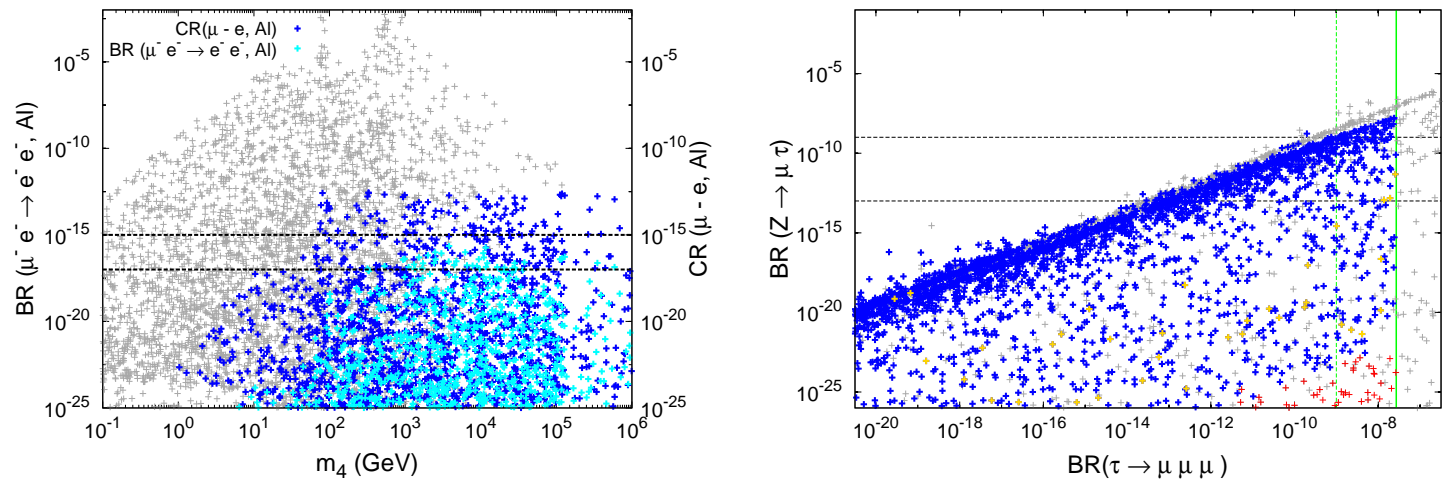

Figure 3: $\mathrm{BR}\left(\mu^{-} e^{-} \rightarrow e^{-} e^{-}, \mathrm{Al}\right)$ (cyan, left axis) and $\mathrm{CR}(\mu-e, \mathrm{Al})$ (dark blue, right axis) as a function of $m_{4}$. Grey points correspond to the violation of at least one experimental bound; dashed horizontal lines denote the future sensitivity of COMET; from [7]. On the right, $\operatorname{BR}(Z \rightarrow \tau \mu)$ vs. $\operatorname{BR}(\tau \rightarrow 3 \mu)$; blue (grey) points denote allowed (excluded) regimes, and yellow points are associated with $0 \vee 2 \beta$ decays within future sensitivity; the upper (lower) horizontal line corresponds to the expected sensitivity for a Linear Collider (FCC-ee), while vertical lines denote current and future $\tau \rightarrow 3 \mu$ sensitivities; from [\&].

\subsection{Mechanisms of neutrino mass generation}

In its different realisations, the seesaw mechanism is perhaps one the most appealing mechanisms of neutrino mass generation. Whether or not a given seesaw realisation can be at the origin of a high-intensity observable depends on the size of the Yukawa-like couplings, and most importantly, on the scale of the new (heavy) mediators. The low-scale seesaw (and its variants) is an example of a type I seesaw, whose mediators have non-negligible mixings with the active neutrinos, and do not decouple. Not only can they give rise to contributions to numerous cLFV observables 
(within future sensitivity reach), but the high-intensity searches for the latter allow to explore and constrain regions of the parameter space which would be otherwise inaccessible [9] (see left panel of Fig. (4).
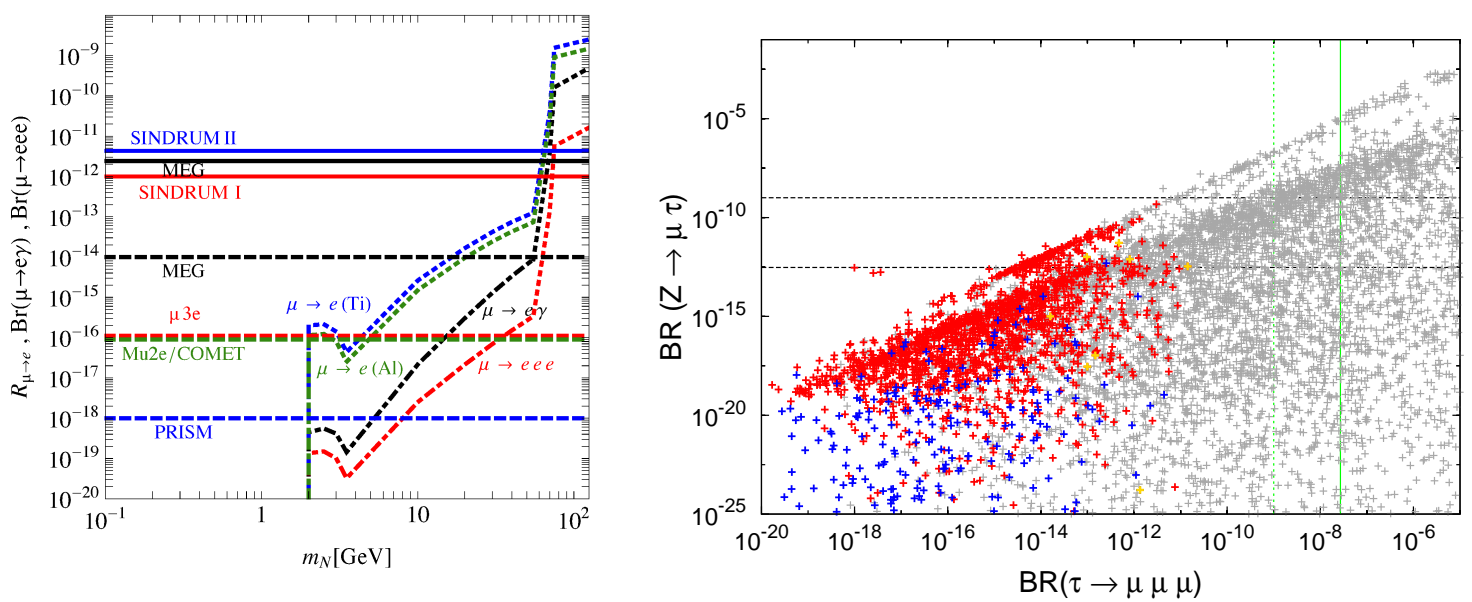

Figure 4: On the left, maximal allowed cLFV rates compatible with current searches in a low-scale seesaw; horizontal full (dashed) lines denote present (future) experimental sensitivity; from [6]. On the right, $\mathrm{BR}(Z \rightarrow \tau \mu)$ vs. $\mathrm{BR}(\tau \rightarrow 3 \mu)$ in a $(3,3)$ ISS realisation; coloured (grey) points denote allowed (excluded) regimes; the upper (lower) horizontal line corresponds to the expected sensitivity for a Linear Collider (FCCee), while vertical lines denote current and future $\tau \rightarrow 3 \mu$ sensitivities; from [8].

Another phenomenologically and theoretically appealing low-scale model of neutrino mass generation is the Inverse Seesaw (ISS). In its $(3,3)$ realisation, three sets of right-handed neutrinos and extra sterile fermions are added to the SM content ${ }^{2}$; the new states do not decouple, leading to modified leptonic currents and extensive contributions to many observables. For example, this is the case of cLFV muonic channels. However, and although $Z \rightarrow \tau \mu$ decays are still within FCCee reach, $\tau \rightarrow 3 \mu$ lies clearly beyond the reach of Belle II (cf. right panel of Fig. $\bigoplus$ ). Although the ISS encompasses several Dirac and Majorana CPV phases, having the heavy states forming pseudo-Dirac pairs precludes significant contributions to lepton EDMs [10].

Due to the triplet nature of the mediators, both type II and type III seesaws lead to very distinctive cLFV signatures. While in all type I-like realisations, cLFV are higher order (loop) processes, in the type II seesaw 3-body decays occur at tree-level; in the type III, both 3-body decays and coherent conversion in Nuclei are tree-level processes (only radiative decays occur at loop level). By constructing ratios of observables, one can aim at disentangling the different realisations: for example, $\operatorname{BR}(\mu \rightarrow e \gamma) / \operatorname{BR}(\mu \rightarrow 3 e) \sim 10^{-3}(\gtrsim 1)$ for type III (I); likewise $\mathrm{CR}(\mu-e, \mathrm{Ti}) / \mathrm{BR}(\mu \rightarrow e \gamma)$ $\sim 10^{3}(\in[0.05-5])$ for type III (II) [11].

\section{Embedding the seesaw in complete NP frameworks}

Aiming at addressing other observational (and theoretical) problems of the SM, the seesaw

\footnotetext{
${ }^{2}$ Since in the ISS the light neutrino masses receive an extra suppression factor from the parameter which is at the origin of all LNV in the model $\left(\mu_{X}\right)$, one can accommodate oscillation data with sizeable Yukawa couplings and a comparatively light NP scale by taking small values of $\mu_{x}$. The model remains theoretically natural, as in the limit $\mu_{x} \rightarrow 0$ one recovers lepton number conservation.
} 
can be embedded in larger, complete NP frameworks, as is the case of supersymmetry (SUSY) or grand unified theories (GUTs).

\subsection{SUSY seesaw}

The SUSY seesaw consists in the embedding of a (for example type I) seesaw in the framework of otherwise flavour conserving SUSY models. Having a unique source of LFV (the neutrino Yukawa couplings) implies that all observables exhibit a strong degree of correlation. This is manifest at low-energies in the strong synergy between $\mu \rightarrow e \gamma$ and $\tau \rightarrow \mu \gamma$ decays, which remain tightly correlated regardless of the typical SUSY spectrum or of the seesaw scale [12] - see left panel of Fig. 5. One can further explore the synergy between low- and high-energy cLFV observables (for instance new edges in dilepton mass distributions, or relative mass differences between left-handed selectrons and smuons [13]) to probe the SUSY seesaw hypothesis. Isolated cLFV manifestations would disfavour the latter, while compatible ones - as for example $\Delta m_{\tilde{\ell}} / m_{\tilde{\ell}}\left(\tilde{e}_{L}, \tilde{\mu}_{L}\right) \gtrsim 0.5 \%$ and an observation of $\mu \rightarrow e \gamma$ at MEG - would not only strengthen it, but further hint on the seesaw scale $\left(M_{R} \sim \mathscr{O}\left(10^{14} \mathrm{GeV}\right)\right)$ [14], as visible on the right panel of Fig. 5.
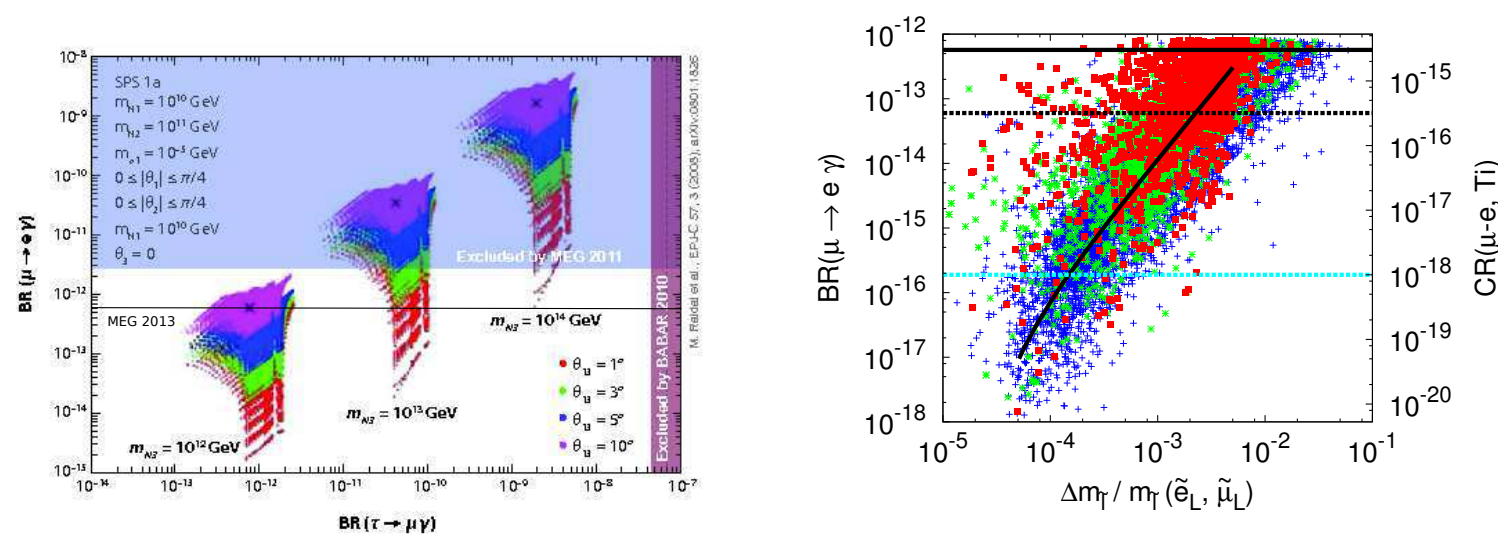

Figure 5: Type I SUSY seesaw: on the left, correlation between $\operatorname{BR}(\mu \rightarrow e \gamma)$ and $\operatorname{BR}(\tau \rightarrow \mu \gamma)$ for different seesaw scales, $M_{R}$; from [12]. On the right, $\operatorname{BR}(\mu \rightarrow e \gamma)$ and $\operatorname{CR}(\mu-e, \mathrm{Ti})$ vs. $\Delta_{\tilde{\ell}} / m_{\tilde{\ell}}\left(\tilde{e}_{L}, \tilde{\mu}_{L}\right)$, also for different seesaw scales, $M_{R}$; from [14].

Increasing the degree of symmetry (be it in the form of extended gauge symmetries, flavour ones, or gauge unification) reduces the arbitrariness of the couplings, rendering the model more predictive, and hence easier to test (and falsify). GUTs are particularly appealing and well-motivated theoretical constructions: in addition to offering a common scheme for Yukawa couplings, they can even relate observables in the lepton and quark sectors. In the simple case of a SU(5) type I SUSY seesaw, there is a strong correlation between flavour violating observables - as well as CP violating observables - in leptons and hadrons (see, for example [15, 16]). A second example of GUT-induced correlation of high-intensity observables can be found in a leptogenesis motivated SO(10) type II SUSY seesaw, which could be easily falsified by any future observation of two low-energy cLFV processes.

\subsection{Further examples: vector-like leptons}

Massive vector-like fermions are present in many well-motivated SM extensions (as is the case 
of composite Higgs, warped extra dimensions, ...). The prospects for cLFV (at high-intensities and in Higgs decays) were addressed in [17], for a generic set-up - inspired by composite Higgs models - in which 3 generations of vector-like left-handed $\left(L_{i}^{V}\right)$ and right-handed $\left(E_{i}^{V}\right)$ charged leptons were included. Neutrino masses can be obtained from additional right-handed states, and the corresponding vector-like partners. The contributions to cLFV observables (and lepton dipole moments) turn out to be parametrised by a small set of couplings, leading to correlated observables. For example one has $\mathrm{BR}\left(h \rightarrow \ell_{i} \ell_{j}\right) / \mathrm{BR}\left(\ell_{i} \rightarrow \ell_{j} \gamma\right) \approx 4 \pi /\left.3 \alpha \mathrm{BR}\left(h \rightarrow \ell_{i} \ell_{i}\right)\right|_{\mathrm{SM}} / \mathrm{BR}\left(\ell_{i} \rightarrow \ell_{j} v_{i} \bar{v}_{j}\right)$. Other than the latter synergy, a strong correlation between EDMs and the muon anomalous magnetic moment $\left(\delta a_{\mu}\right)$ was also found. Interestingly, attempts to explain the current tension between theory and observation in $(g-2)_{\mu}$ implies excessive contributions to the electron EDM, almost leading to the exclusion of the model - as can be seen from Fig. 6 (left panel).
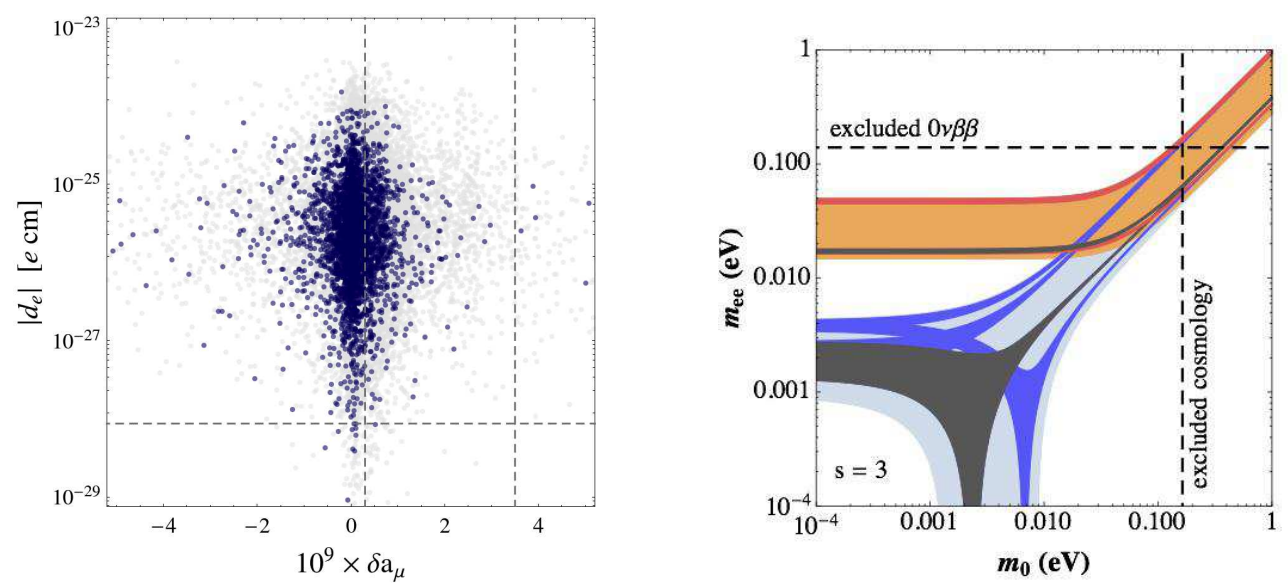

Figure 6: On the left, correlation of $\delta a_{\mu}$ with the electron EDM for a model of vector-like leptons; grey points are ruled out by LHC, and dashed lines show the $2 \sigma$ experimental region for $\delta a_{\mu}$, and the 90\% C.L. upper bound on $\left|d_{e}\right|$; from [17]. On the right, effective Majorana mass $m_{e e}$ as a function of the lightest neutrino mass, for flavour groups $\Delta\left(3 n^{2}\right)$ and specific classes of CP transformations; from [19].

\subsection{Further examples: flavour symmetries and massive neutrinos}

The flavour puzzle remains one of the most important open questions in particle phyics. A possible way to address it, starting from first principles, it to relate the flavour patterns (for example, the texture of the Yukawa couplings) to the breaking of a flavour symmetry $G_{f}$, continuous or discrete. This avenue has been extensively explored in recent years, relying on very distinct approaches. The only phenomenological caveat of certain constructions lies on the difficulty of testing them - however, many realisations have well-defined, peculiar signatures. We have discussed two illustrative examples: (i) continuous flavour symmetry - minimal Abelian case, with $G_{f}=\mathrm{U}(1)_{L_{e}+L_{\mu}} \times \mathrm{U}(1)_{L_{\tau}}$ leading to predictions of the $\mathrm{BR}(\mu \rightarrow e \gamma)$ correlated with the ordering scheme of the light neutrino spectrum (for an example see [18]); (ii) a discrete group based approach, with $G_{f}$ of the $\Delta\left(3 n^{2}\right)$ type, which predicts both lepton mixings as well as low- and high-scale CPV phases [19] (see Fig. 6). Other than constraining predictions for neutrinoless double beta decays, the latter construction further leads to the interplay of low-energy CP phases and a successful explanation of the baryon asymmetry of the Universe from leptogenesis. 


\section{Overview}

While remaining one of the most important open questions in modern particle physics, astrophysics and cosmology, neutrinos have proved to be true gateways to numerous new physics phenomena. An extensive number of dedicated facilities is devoted to searching for the latter, and the near future should see new data that will hopefully clarify several points.

Currently, a number of confirmed observations and several tensions between experimental data and SM expectations suggests the need to consider NP scenarios; interestingly, many of these tensions are nested in lepton related-observables. We have briefly overviewed a small subset of high-intensity observables ${ }^{3}$, and the potential contributions of a few NP models, in particular of realisations aiming at addressing the problem of neutrino mass generation. We have also discussed several examples of how the synergy between neutrino data and searches at the high-intensity frontier might provide information on the underlying NP model of neutrino mass generation.

\section{References}

[1] Proceedings of "The 19th International Workshop on Neutrinos from Accelerators (NUFACT2017)", held 25-30 September 2017 at Uppsala University.

[2] C. Patrignani et al. [Particle Data Group], Chin. Phys. C 40 (2016) no.10, 100001.

[3] G. M. Pruna, these proceedings (WG4); A. De Gouvea, these proceedings.

[4] A. Abada and T. Toma, JHEP 1602 (2016) 174.

[5] C. Giunti, Nucl. Phys. B 908 (2016) 336.

[6] A. Abada, V. De Romeri, M. Lucente, A. M. Teixeira and T. Toma, "Effective Majorana mass matrix from tau and pseudoscalar meson lepton number violating decays,” arXiv:1712.03984 [hep-ph].

[7] A. Abada, V. De Romeri and A. M. Teixeira, JHEP 1602 (2016) 083.

[8] A. Abada, V. De Romeri, S. Monteil, J. Orloff and A. M. Teixeira, JHEP 1504 (2015) 051.

[9] R. Alonso et al, JHEP 1301 (2013) 118.

[10] A. Abada and T. Toma, JHEP 1608 (2016) 079.

[11] T. Hambye, Nucl. Phys. Proc. Suppl. 248-250 (2014) 13.

[12] S. Antusch, E. Arganda, M. J. Herrero and A. M. Teixeira, JHEP 0611 (2006) 090.

[13] A. Abada, A. J. R. Figueiredo, J. C. Romao and A. M. Teixeira, JHEP 1010 (2010) 104.

[14] A. J. R. Figueiredo and A. M. Teixeira, JHEP 1401 (2014) 015.

[15] L. Calibbi et al, JHEP 0912 (2009) 057.

[16] A. J. Buras, M. Nagai and P. Paradisi, JHEP 1105 (2011) 005.

[17] A. Falkowski, D. M. Straub and A. Vicente, JHEP 1405 (2014) 092.

[18] F. F. Deppisch, Fortsch. Phys. 61 (2013) 622.

[19] C. Hagedorn and E. Molinaro, Nucl. Phys. B 919 (2017) 404.

[20] M. Yamanaka, these proceedings; see also A. Abada, V. De Romeri, J. Orloff and A. M. Teixeira, Eur. Phys. J. C 77 (2017) no.5, 304.

\footnotetext{
${ }^{3}$ Further interesting observables include the Muonium system [7] or "in-flight cLFV conversion" [20], among others.
} 